\title{
Evaluation of subgrid-scale models in large-eddy simulation of flow past a two-dimensional block
}

\author{
Wai-Chi Cheng, Fernando Porté-Agel* \\ Wind Engineering and Renewable Energy Laboratory (WIRE), École Polytechnique Fédérale de Lausanne (EPFL), EPFL-ENAC-IIE-WIRE, 1015 Lausanne, Switzerland
}

\section{A R T I C L E I N F O}

\section{Article history:}

Received 18 January 2013

Received in revised form 23 June 2013

Accepted 24 June 2013

Available online 20 July 2013

\section{Keywords:}

Large-eddy simulation

Subgrid-scale models

Immersed boundary method

Two-dimensional block

Turbulence kinetic energy budget

\begin{abstract}
A B S T R A C T
Large-eddy simulations of flow past a two-dimensional (2D) block were performed to evaluate four subgrid-scale (SGS) models: (i) the traditional Smagorinsky model, (ii) the Lagrangian dynamic model, (iii) the Lagrangian scale-dependent dynamic model, and (iv) the modulated gradient model. An immersed boundary method was employed to simulate the $2 \mathrm{D}$ block boundaries on a uniform Cartesian grid. The sensitivity of the simulation results to grid refinement was investigated by using four different grid resolutions. The velocity streamlines and the vertical profiles of the mean velocities and variances were compared with experimental results. The modulated gradient model shows the best overall agreement with the experimental results among the four SGS models. In particular, the flow recirculation, the reattachment position and the vertical profiles are accurately reproduced with a relative coarse grid resolution of $\left(N_{x} \times N_{y} \times N_{z}=\right) 160 \times 40 \times 160\left(n_{x} \times n_{z}=13 \times 16\right.$ covering the block). Besides the modulated gradient model, the Lagrangian scale-dependent dynamic model is also able to give reasonable prediction of the flow statistics with some discrepancies compared with the experimental results. Relatively poor performance by the Lagrangian dynamic model and the Smagorinsky model is observed, with simulated recirculating patterns that differ from the measured ones. Analysis of the turbulence kinetic energy (TKE) budget in this flow shows evidence of a strong production of TKE in the shear layer that forms as the flow is deflected around the block.
\end{abstract}

(c) 2013 Elsevier Inc. All rights reserved.

\section{Introduction}

Atmospheric boundary layer (ABL) flow often develops over complex terrain such as urban areas or mountainous terrain. In this kind of flow, the shape of the terrain surface has a strong effect on the turbulence generation process and the turbulent transport of momentum and scalars (Cai, 1999). In particular, flow separation and recirculation are usually found near the surface and play an important role on the spatial distribution of turbulence kinetic energy (TKE) and turbulent fluxes of momentum and scalars. In order to obtain fundamental understanding on how turbulent boundarylayer flow is affected by the presence of bluff bodies, several studies have been conducted using both experiments and numerical simulations (e.g., Castro and Robins, 1977; Hunt et al., 1990; Hussein and Martinuzzi, 1996; Krajnović, 2009; Lim et al., 2009; Yakhot et al., 2006).

Among the computational methods used in the literature, largeeddy simulation (LES) has become a popular tool to simulate flow past bluff bodies in recent decades. This is mainly due to its ability to explicitly resolve the large-scale turbulence in high-

\footnotetext{
* Corresponding author. Address: EPFL-ENAC-IIE-WIRE, Bâtiment GR, Station 2, 1015 Lausanne, Switzerland. Tel.: +41216932726.

E-mail address: fernando.porte-agel@epfl.ch (F. Porté-Agel).
}

Reynolds-number (Re) flows with nowadays affordable computational resources (e.g., Pope, 2000; Meneveau and Katz, 2000; Sagaut, 2005; Voller and Porté-Agel, 2002). In LES, all the smallscale turbulence which is not resolved is parameterized using a subgrid-scale (SGS) model. In high-Re boundary-layer flows such as the ABL, characterized by a very wide range of eddy scales, LES results are sensitive to the choice of SGS model. This is particularly the case near the surface, where the flow is strongly anisotropic and the subgrid scales account for a large fraction of the turbulent fluxes. Various types of SGS models have been proposed in the literature (Sagaut, 2005), some of which have already been applied to the study of flow past bluff bodies. Most SGS models are based on the eddy-viscosity approach, in which the deviatoric part of the SGS stress is parameterized as

$\tau_{i j}-\frac{1}{3} \delta_{i j} \tau_{k k}=-2 v_{t} \widetilde{S}_{i j}$

where $\tau_{i j}=\widetilde{u_{i} u_{j}}-\tilde{u}_{i} \tilde{u}_{j}$ is the SGS stress, $\widetilde{S}_{i j}=\frac{1}{2}\left(\partial \tilde{u}_{i} / \partial x_{j}+\partial \tilde{u}_{j} / \partial x_{i}\right)$ is the resolved strain-rate tensor, $v_{t}$ is the SGS eddy viscosity, $\delta_{i j}$ is the Kronecker delta, $u_{i}$ is the flow velocity in the $i$-direction (with $i=1,2,3$ corresponding to the streamwise $(x)$, spanwise $(y)$ and vertical $(z)$ directions) and the notation $\tilde{\phi}$ corresponds to the resolved filtered component of the variable $\phi$. The value of $v_{t}$ is modeled by (Smagorinsky, 1963) 
$v_{t}=\left[C_{s} \Delta\right]^{2}|\widetilde{S}|$,

where $\Delta$ is the filter size, $C_{s}$ is the Smagorinsky coefficient and $|\widetilde{S}|$ is the magnitude of $\widetilde{S}_{i j}$. For the traditional Smagorinsky model, $C_{s}$ is considered as constant. The traditional Smagorinsky model was used by Murakami et al. (1987) to study the flow around a cube at Reynolds number of $10^{5}$, and two values of $C_{s}=0.1$ and 0.2 were used. The simulation results showed that the resolved turbulence intensity is very sensitive to the near-wall treatment and the value of the Smagorinsky constant. Some small-scale velocity fluctuations can only be observed when using a small Smagorinsky constant value. Later, the dynamic model (Germano et al., 1991) was used to simulate the turbulent flow past a cubic obstacle (Shah and Ferziger, 1997) at Reynolds number of $3 \times 10^{4}$ and $4 \times 10^{4}$. Instead of a constant $C_{s}$, the dynamic model calculates the value of the Smagorinsky coefficient locally by minimizing the error made when computing the resolved Leonard stress (defined using a test-filter scale, typically of size 24) using the Smagorinsky model and assuming scale invariance of the model coefficient (more details are given by Germano et al. (1991)) The mean wind and turbulence profiles, the positions of flow separation, the reattachment length and the turbulence coherent structure were considered in Shah and Ferziger (1997). The results suggest that careful choice of the SGS model and numerical methods are necessary to reproduce the real flow structure. By considering the flow over a bluff rectangular plate at Reynolds number of $5 \times 10^{4}$, Suksangpanomrung et al. (2000) evaluated three SGS models: (i) The standard Smagorinsky model, (ii) the structure function model, and (iii) the selective structure function model. In the structure function model, the value of $v_{t}$ is directly proportional to the square root of the second-order structure function of the resolved velocity field. The selective structure function model further constraints the eddy viscosity to be nonzero only when the flow is sufficiently three-dimensional, and it was found to be the most accurate model among the three models tested. Two types of one-equation models were also used by Krajnović and Davidson (2002) in simulations of flow around a surfacemounted cube at Reynolds number of $4 \times 10^{4}$. Both of them give reasonable prediction of the separation region and reattachment length.

In this study, we test the performance of the Lagrangian scaledependent dynamic model (Porté-Agel et al., 2000; Stoll and Porté-Agel, 2006a) and the modulated gradient model (Lu and Porté-Agel, 2010) to study the flow past a two-dimensional (2D) block. This validation case is chosen because $2 \mathrm{D}$ block is commonly considered as an idealized building due to its simplicity in geometry, while still possessing the basic characteristics of a building block. This makes it an ideal case for testing the performance of LES models in simulations of flow in urban environment. The wind tunnel experimental data of Baker (1977) and Moss and Baker (1980) is used since several vertical profiles of mean wind velocity and velocity variance are available for comparison.

The scale-dependent dynamic model (Porté-Agel et al., 2000) was developed in order to generalize the standard dynamic model to include scale dependence. To achieve this, a secondary test filter is applied to determine the scale-dependence factor $\beta \equiv C_{s}^{2}(2 \Delta) / C_{s}^{2}(\Delta)$. The development of the scale-dependent dynamic model is in view of the assumption of scale invariance may not always hold as it requires the existence of an idealized inertial range and the filtering scale falling within that range. It was used to simulate neutral homogeneous ABL flow (Porté-Agel et al., 2000) and scalar transport (Porté-Agel, 2004), and improved results over the Smagorinsky and standard dynamic models were observed. Later, Stoll and Porté-Agel (2006a), Wan and Porté-Agel (2011a,b), and Wan et al. (2007) used the scale-dependent dynamic model together with Lagrangian averaging (Meneveau et al., 1996) to simulate ABL flow over heterogeneous terrain. With the Lagrangian approach, averaging over flow pathlines is performed in the calculation of the Smagorinsky coefficient. This allows the calculated Smagorinsky coefficient to adjust to the horizontal flow inhomogeneities. Other than the Lagrangian scale-dependent dynamic model, the Lagrangian dynamic model (Meneveau et al., 1996) is also tested in this study to simulate flow past a 2D block.

The other SGS model that is evaluated is the recently-developed modulated gradient model (Lu and Porté-Agel, 2010). In simulations of a neutral ABL, it is able to capture the most important statistical characteristics of boundary-layer turbulence. In this model, the SGS stress is calculated as

$\tau_{i j}=2 k_{s g s}\left(\frac{\widetilde{G}_{i j}}{\widetilde{G}_{k k}}\right)$,

where $k_{s g s}$ is the SGS kinetic energy and $\widetilde{G}_{i j}$ is obtained by Taylor's expansion of $\tau_{i j}$

$\widetilde{G}_{i j}=\frac{\Delta_{x}^{2}}{12} \frac{\partial \tilde{u}_{i}}{\partial x} \frac{\partial \tilde{u}_{j}}{\partial x}+\frac{\Delta_{y}^{2}}{12} \frac{\partial \tilde{u}_{i}}{\partial y} \frac{\partial \tilde{u}_{j}}{\partial y}+\frac{\Delta_{z}^{2}}{12} \frac{\partial \tilde{u}_{i}}{\partial z} \frac{\partial \tilde{u}_{j}}{\partial z}$,

where $\Delta_{x}, \Delta_{y}$ and $\Delta_{z}$ correspond to the streamwise, spanwise and vertical grid spacing, respectively. The value of $k_{s g s}$ is calculated with the assumption of local equilibrium (Lu and Porté-Agel, 2010)

$k_{s g s}=\frac{4 \Delta^{2}}{C_{\epsilon}^{2}}\left(-\frac{\widetilde{G}_{i j}}{\widetilde{G}_{k k}} \widetilde{S}_{i j}\right)^{2}$,

with $C_{\epsilon} \approx 1$ based on the assumption of an averaged energy balance between SGS energy production and molecular dissipation rate. The main advantages of the modulated gradient model are: (i) the model satisfies material frame indifference, (ii) it is simple and computationally inexpensive as no test filtering or additional transport equation is needed, and (iii) it is able to capture flow anisotropy better than eddy-viscosity models. As mentioned in Lu and PortéAgel (2010), the eddy-viscosity closure assumes a one-to-one correlation between the SGS stress tensor and the strain rate tensor, and locally employs the same eddy-viscosity for all directions. This may be inaccurate and could induce errors when simulating strong anisotropic flows like the one considered here. Therefore, it is of interest to test and compare the performance of the modulated gradient model with the existing eddy-viscosity models.

\section{Numerical setup}

LESs were performed of flow past a 2D block with height $h$ and width $2 h$. The numerical setup was constructed to reproduce the conditions of the wind tunnel experiment of Baker (1977) and Moss and Baker (1980). Simulation and experimental results were compared to evaluate four SGS models: the Smagorinsky model, the Lagrangian dynamic model, the Lagrangian scale-dependent dynamic model and the modulated gradient model.

The computational domain is shown in Fig. 1 with dimensions $\left(L_{x} \times L_{y} \times L_{z}\right)$ equal to $25 h \times 6.25 h \times 10 h$. Periodic boundary conditions were used in the lateral directions and the top boundary was considered as a stress-free wall, while the bottom boundary was a rough wall. A buffer zone was adopted to smoothly adjust the flow from the far wake to a uniform inflow condition with constant wind speed $U_{0}$. The length of the buffer zone equals to $1 \mathrm{~h}$ is used so that it is long enough to ensure there is no sharp velocity change within the buffer zone which would induce numerical oscillation in the simulation results. In addition, the buffer zone is located at $5 \mathrm{~h}$ upstream of the block with the aim to reproduce the same physical inflow conditions as those of the experiment. Laminar inflow condition is used as in the experiment. As the flow comes out from the inlet, a thin boundary layer grows near ground surface along the streamwise direction. 


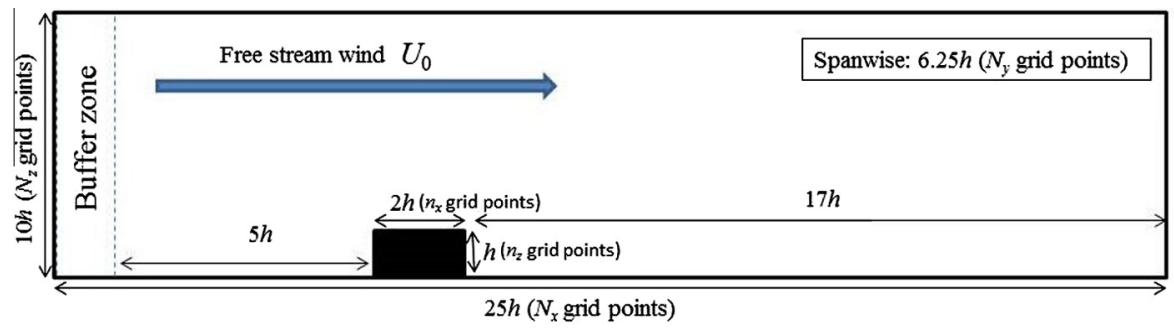

Fig. 1. The computational domain.

The use of similar buffer zone in a pseudospectral LES code to impose the inflow boundary condition has been successful in former studies of flow through urban canopy (Tseng et al., 2006), a steep hill (Wan and Porté-Agel, 2011a), a wind turbine (Porté-Agel et al., 2011; Wu and Porté-Agel, 2011, 2012), and wind farms (Wu and Porté-Agel, 2013).

The LES code solves the filtered continuity equation and the filtered incompressible Navier-Stokes equations in rotational form (Orszag and Pao, 1975):

$$
\begin{aligned}
& \frac{\partial \tilde{u}_{i}}{\partial x_{i}}=0 \\
& \frac{\partial \tilde{u}_{i}}{\partial t}+\tilde{u}_{j}\left(\frac{\partial \tilde{u}_{i}}{\partial x_{j}}-\frac{\partial \tilde{u}_{j}}{\partial x_{i}}\right)=-\frac{\partial \tilde{p}^{*}}{\partial x_{i}}-\frac{\partial \tau_{i j}}{\partial x_{j}}+v \frac{\partial^{2} \tilde{u}_{i}}{\partial x_{j}^{2}}+f_{i},
\end{aligned}
$$

where $\tilde{u}_{i}$ is the filtered velocity in the $i$ direction, $\tilde{p}^{*}=\tilde{p} / \rho+\frac{1}{2} \tilde{u}_{i} \tilde{u}_{i}$ is the modified kinematic pressure, $\tau_{i j}$ is the SGS stress tensor, and $v$ is the kinematic viscosity of air. The term $f_{i}$ is the immersed forcing used to simulate the effect of the block on the flow. The simulation results are compared with results of the wind tunnel experiment (Moss and Baker, 1980) with Re, based on the main stream velocity and step height, approximately equal to $5 \times 10^{4}$. The experiment was conducted under neutral conditions and therefore no additional term was used to account for the effect of buoyancy in Eq. (7).

The bottom boundary condition is based on the logarithmic wind profile (Businger et al., 1971). Although the theory is only valid for averaged quantities under steady and homogeneous conditions, it is commonly used also to fluctuating (LES filtered) quantities in both homogeneous and heterogeneous flows. Eventhough recent studies have highlighted the limitations of this approach (e.g., Marusic et al., 2001; Chamorro and Porté-Agel, 2010; Abkar and Porté-Agel, 2012), no alternative is available for complex flows. Under neutral conditions, the surface shear stress $\tau_{i 3, s}(x, y, t)(i=1,2)$ is often computed as (Stoll and Porté-Agel, $2008,2006 b)$

$\tau_{i 3, s}(x, y, t)=-\left[\frac{\kappa \tilde{u}_{r}(x, y, z, t)}{\ln \left(z / z_{0}\right)}\right]^{2} \frac{\tilde{u}_{i}(x, y, z, t)}{\tilde{u}_{r}(x, y, z, t)}$,

where the subscript $s$ denotes surface values, $\tilde{u}_{r}(x, y, z, t)=$ $\left[\tilde{u}_{1}(x, y, z, t)^{2}+\tilde{u}_{2}(x, y, z, t)^{2}\right]^{1 / 2}$ is the local instantaneous (filtered) horizontal velocity magnitude at height $z=\Delta_{z} / 2, \kappa$ is the von Kármán constant, and $z_{0}=10^{-3} \mathrm{~h}$ is the aerodynamic surface roughness, which is estimated base on the relation $z_{0}=0.12 v / u_{*}$ for dynamically smooth surface (Hinze, 1975) with $u_{*}$ calculated using the experimental data. A simple sensitivity test on $z_{0}$ was performed with simulations using $z_{0}$ equal to $2 \times 10^{-3} h, 10^{-3} h$ and $5 \times 10^{-4} h$. No significant change in the simulation results (velocity profiles and recirculation pattern) was observed.

Note that, although laminar inflow condition is currently used at the inlet, there is a thin turbulent boundary layer developing along the streamwise direction upstream of the 2D block. Therefore, the same surface boundary condition is still used there.
The spatial derivatives in the horizontal directions were calculated based on the pseudo-spectral method, while the second-order central-difference method was used in the vertical direction. More details on the LES code can be found in Albertson (1996), Porté-Agel (2004, 2000), Stoll and Porté-Agel (2008, 2006b).

An immersed boundary (IB) method (Mittal and Iaccarino, 2005) was used to model the presence of the bluff body by specifying an immersed forcing term $f_{i}$ in Eq. (7). A Cartesian grid with staggered arrangement was used. The levels of computation for the vertical velocity $\tilde{w}$ are half-grid shifted compared with the computational levels for the streamwise velocity $\tilde{u}$, spanwise velocity $\tilde{v}$, and $\tilde{p}^{*}$. The vertical surfaces of the block are located at the middle of two grid columns while the top surface is located at the computational level of $\tilde{w}$ (Fig. 2). At the block surface, the surface shear stress was computed using a method similar to the one used for the bottom surface, i.e. applying the logarithmic wind profile to the fluctuating quantities using the local instantaneous velocity components that are parallel to the wall. Moreover, smoothing of the velocity field inside the block before the velocity derivatives calculation was used to diminish the Gibbs phenomenon that occurs near sharp boundaries (Fang et al., 2011; Tseng et al., 2006). The smoothing was performed in the streamwise direction based on a second-order polynomial equation.

\section{Results}

In the following discussion of the LES results, spanwise- and time-averaged (denoted by $\langle\cdot\rangle$ ) values of the flow variables are presented. The statistics are computed over more than 40 times the time required for the flow to go through the entire domain $\left(\approx 1000 h / U_{0}\right)$ after achieving quasi-steady flow conditions. The filtered velocity $\tilde{u}_{i}$ is decomposed into the average $\left\langle\tilde{u}_{i}\right\rangle$ and fluctuation $\tilde{u}_{i}^{\prime}$ components as

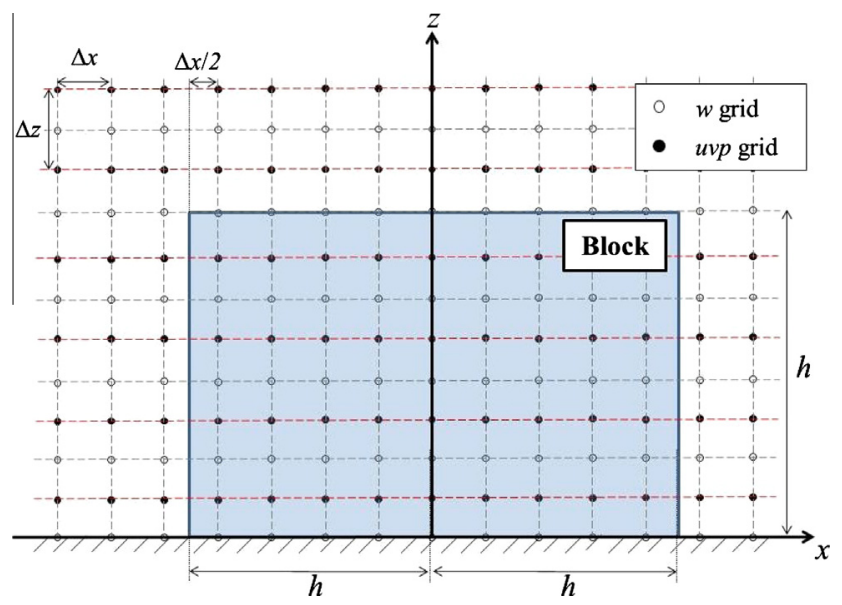

Fig. 2. The grid configuration of the immersed boundary (IB) method. 
$\tilde{u}_{i}=\left\langle\tilde{u}_{i}\right\rangle+\tilde{u}_{i}^{\prime}$

\subsection{Flow patterns and grid resolution tests}

Four grid resolutions of $N_{x} \times N_{y} \times N_{z}=200 \times 50 \times 200$, $160 \times 40 \times 160,120 \times 30 \times 120$ and $80 \times 20 \times 80$ were tested, which correspond to a number of grid points covering the block of $\left(n_{x} \times n_{z}=\right) 16 \times 20,13 \times 16,10 \times 12$ and $6 \times 8$, respectively. The wind tunnel experimental results (Moss and Baker, 1980) showed that as the incoming flow is deflected by the 2D block, flow separation occurs and several recirculation regions form. In particular, one big recirculation zone is observed behind the block while two small recirculations are located at the two corners formed by the ground and the vertical boundaries of the block (Fig. 3). The big recirculation forms as a result of the flow separation occurring at the top upwind corner of the block; the separated flow then reattaches at a downstream position of about $10 \mathrm{~h}$ from the downwind wall.

Velocity streamlines obtained from the results of the four SGS models with different grid resolutions are shown in Fig. 4. In all the simulations, a big recirculation zone is observed. The locations of the reattachment position and the big recirculation center for all the cases are reported in Table 1 . The results from the modulated gradient model show the best overall agreement with the measurements among all the SGS models. For the modulated gradient model, grid independent results are achieved at the grid resolution of $160 \times 40 \times 160$ as nearly the same flow statistics are obtained as those from the finer grid resolution of $200 \times 50 \times 200$. The big recirculation zone is accurately reproduced as shown in Table 1. Specifically, the locations of the reattachment $(x / h=10.7)$ and the big recirculation center $(x / h=4.5, z / h=1.0)$ predicted with the modulated gradient model (with grid resolution of $160 \times 40 \times 160)$ agree very well with the experimental results $(x / h=10.8$ and $x / h=4.6, z / h=1.1$, respectively). The small recirculation located downstream of the block is also well predicted by the modulated gradient model with similar size to that measured in the experiment. The main discrepancy between the numerical and the experimental results is that the simulated small recirculation upstream of the block is flatter than the measured one. Reasonable results are still obtained with resolution of $120 \times 30 \times 120$, at which a higher and larger big recirculation zone is formed. With the lowest resolution of $80 \times 20 \times 80$, the model is not able to resolve the recirculations accurately, although the size of the big recirculation zone is still comparable with the measurements. At that resolution, large differences between simulations and experiments are found in the sizes and shapes of the two small recirculations.

Other than the modulated gradient model, the Lagrangian scaledependent dynamic model is also able to accurately reproduce the recirculation pattern with grid resolutions of $160 \times 40 \times 160$ and $200 \times 50 \times 200$, at which the predicted locations of the reattachment point and the recirculation center agree well with the experimental data (Table 1 ). The two small recirculations are reproduced by the Lagrangian scale-dependent dynamic model with sizes that agree with the experimental results. Some differences between the results of the two resolutions $200 \times 50 \times 200$ and $160 \times 40 \times 160$ are observed. As shown in Table 1, the flow reattaches at a shorter distance $(x / h=10.2)$ in the simulation with grid resolution of $200 \times 50 \times 200$ compared with the one with grid resolution of $160 \times 40 \times 160$, for which reattachment occurs at $x / h=10.8$. Fairly reasonable results with the Lagrangian scale-dependent dynamic model are also obtained with grid resolution of $120 \times 30 \times 120$. However, the center of the big recirculation is found at a further downstream position $(x / h=5.2, z / h=1.1)$ than that observed in the experiment. Moreover, the two small recirculations are not well resolved with this resolution. With the lowest grid resolution of $80 \times 20 \times 80$, the small recirculation upstream of the block is not resolved at all, while the one located downstream is too big compared with the measured one. Moreover, the big recirculation is also not well resolved, and oscillations are observed in the simulated velocity streamlines.

The Lagrangian dynamic model yields a big recirculation region behind the block that is too small for the finer grid resolutions of $200 \times 50 \times 200$ and $160 \times 40 \times 160$. Consistent with that result, the flow reattaches too close to the block for those resolutions, at $x / h=8.1$ and 9.6, respectively (Table 1 ). Both small recirculations are reproduced in the simulations, with the one near the upwind wall having a slightly larger size than that observed in the experiment. Also shown in Table 1, the positions of the reattachment and the recirculation center vary a lot between the simulations with grid resolutions of $200 \times 50 \times 200$ and $160 \times 40 \times 160$, which show the strong resolution dependence of the results from the Lagrangian dynamic model. Fairly reasonable results are obtained with the resolution of $120 \times 30 \times 120$, with all recirculation zones resolved, but with sizes different from the measurements. For the lowest resolution of $80 \times 20 \times 80$, the three recirculation zones are poorly resolved. Oscillations in the velocity streamlines are also observed in the big recirculation zone.

For the Smagorinsky model, simulations with $C_{s}=0.1$ and 0.2 are tested. The three recirculation zones are fairly well reproduced in the simulations with grid resolutions of $200 \times 50 \times 200$ and $160 \times 40 \times 160$. However, the simulation results are found to be very sensitive to the values of $C_{s}$ used. As shown in Fig. 4 and Table 1 , the recirculation center of the big recirculation simulated with $C_{s}=0.2$ is located more downstream than the one simulated with $C_{s}=0.1$. In particular, in the simulations with grid resolutions of $200 \times 50 \times 200$ and $160 \times 40 \times 160$, the recirculation center changes from $x / h=4.0$ to 5.0 and from $x / h=4.0$ to 5.8 , respectively, as $C_{s}$ changes from 0.1 to 0.2 . Moreover, the small recirculation near the downwind corner becomes too big in the simulation with $C_{s}=0.2$. For the simulations with lower resolutions of $120 \times 30 \times 120$ and $80 \times 20 \times 80$, the differences between the simulation results with the two $C_{s}$ become more obvious. For the simulations with $C_{s}=0.1$, the recirculation center is found at $x / h=3.8$ and 4.3 with grid resolutions of $120 \times 30 \times 120$ and $80 \times 20 \times 80$, respectively. In both cases, the recirculation center is shifted upstream with respect to its measured position of $x / h=4.6$. Interestingly, in the simulations with $C_{s}=0.2$ and grid

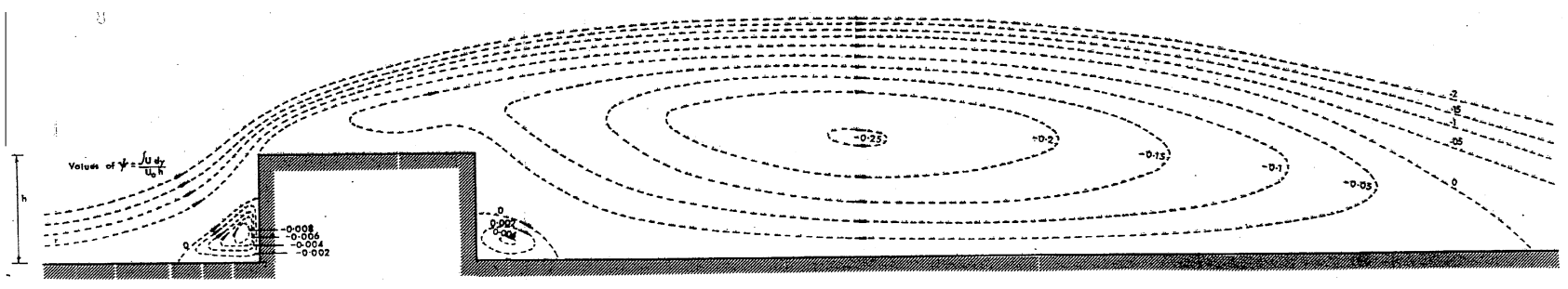

Fig. 3. Velocity streamlines from Moss and Baker (1980). 


\section{Moss and Baker (1980)}
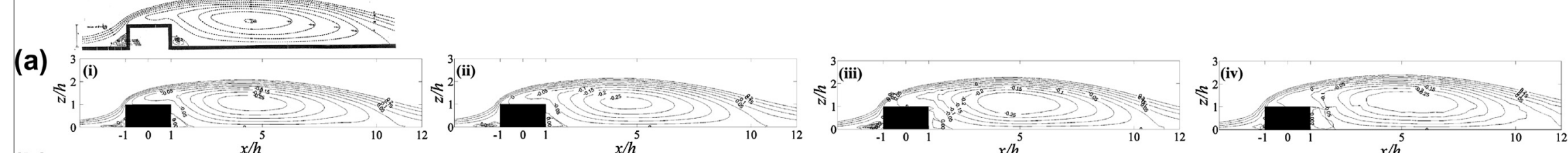

\section{(b)}
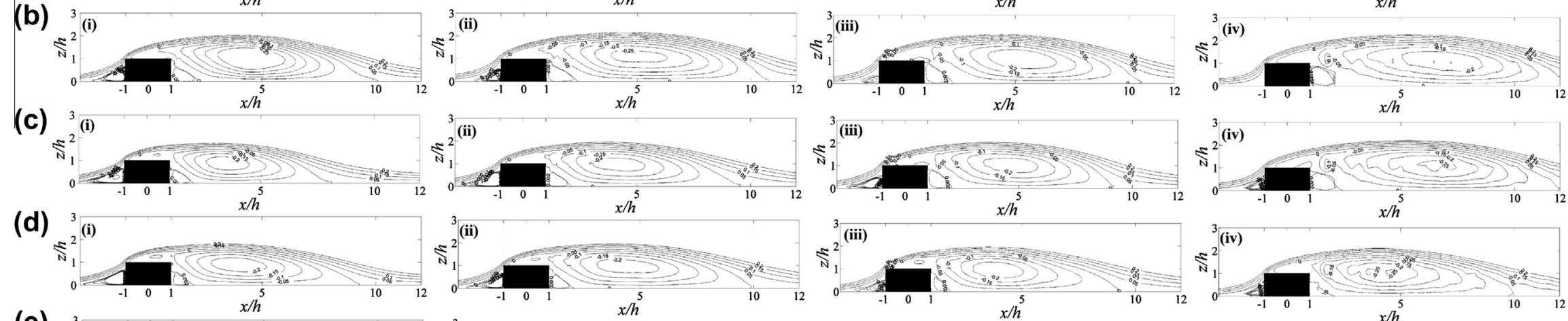

\section{(e)}
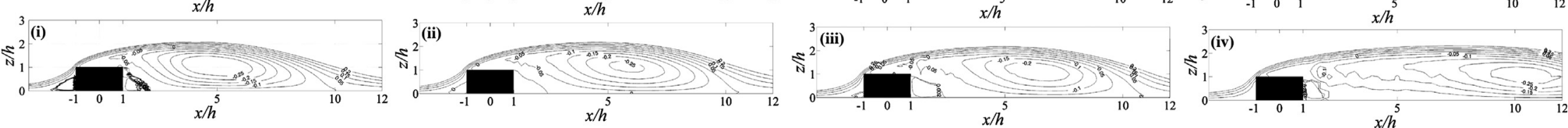

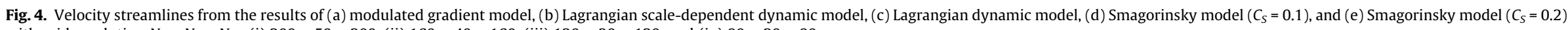
with grid resolution $N_{x} \times N_{y} \times N_{z}=$ (i) $200 \times 50 \times 200$, (ii) $160 \times 40 \times 160$, (iii) $120 \times 30 \times 120$, and (iv) $80 \times 20 \times 80$. 
Table 1

Locations of reattachment position and center of the main recirculation.

\begin{tabular}{|c|c|c|c|}
\hline Model & Grid resolution & Reattachment position $(x / h)$ & Recirculation center $(x / h, z / h)$ \\
\hline Moss and Baker (1980) & & 10.8 & $(4.6,1.1)$ \\
\hline Modulated gradient model & $\begin{array}{r}200 \times 50 \times 200 \\
160 \times 40 \times 160 \\
120 \times 30 \times 120 \\
80 \times 20 \times 80\end{array}$ & $\begin{array}{l}11.0 \\
10.7 \\
11.3 \\
12.3\end{array}$ & $\begin{array}{l}(4.5,1.1) \\
(4.5,1.0) \\
(4.6,1.2) \\
(5.4,1.3)\end{array}$ \\
\hline Lagrangian scale-dependent dynamic model & $\begin{array}{r}200 \times 50 \times 200 \\
160 \times 40 \times 160 \\
120 \times 30 \times 120 \\
80 \times 20 \times 80\end{array}$ & $\begin{array}{l}10.2 \\
10.8 \\
10.5 \\
12.5\end{array}$ & $\begin{array}{l}(4.5,1.1) \\
(4.6,1.1) \\
(5.2,1.1) \\
(7.2,1.2)\end{array}$ \\
\hline Lagrangian dynamic model & $\begin{array}{r}200 \times 50 \times 200 \\
160 \times 40 \times 160 \\
120 \times 30 \times 120 \\
80 \times 20 \times 80\end{array}$ & $\begin{array}{r}8.1 \\
9.6 \\
9.2 \\
12.0\end{array}$ & $\begin{array}{l}(3.3,1.0) \\
(4.5,0.9) \\
(4.9,1.0) \\
(6.8,1.2)\end{array}$ \\
\hline Smagorinsky model $\left(C_{s}=0.1\right)$ & $\begin{array}{r}200 \times 50 \times 200 \\
160 \times 40 \times 160 \\
120 \times 30 \times 120 \\
80 \times 20 \times 80\end{array}$ & $\begin{array}{r}9.5 \\
9.9 \\
9.3 \\
10.0\end{array}$ & $\begin{array}{l}(4.0,1.0) \\
(4.0,1.0) \\
(3.8,1.0) \\
(4.3,1.1)\end{array}$ \\
\hline Smagorinsky model $\left(C_{s}=0.2\right)$ & $\begin{array}{r}200 \times 50 \times 200 \\
160 \times 40 \times 160 \\
120 \times 30 \times 120 \\
80 \times 20 \times 80\end{array}$ & $\begin{array}{l}10.0 \\
10.5 \\
10.9 \\
16.2\end{array}$ & $\begin{array}{l}(5.0,1.1) \\
(5.8,1.1) \\
(6.5,1.1) \\
(10.8,1.0)\end{array}$ \\
\hline
\end{tabular}

resolutions of $120 \times 30 \times 120$ and $80 \times 20 \times 80$, the simulated big recirculation zones are much longer and have their centers at $x /$ $h=6.5$ and 10.8, respectively. The big differences in the simulation results from the Smagorinsky model with $C_{s}=0.1$ and 0.2 demonstrate a well-known limitation of the standard Smagorinsky model in simulations of complex flows: the model is unable to adjust the model coefficient to account for the local variability of the turbulence scale, which makes the results strongly dependent on the choice of $C_{s}$.

The computation times of simulations using the four SGS models with the grid resolution of $160 \times 40 \times 160$ are compared to evaluate the computational costs associated with the different SGS models. With the same number of time steps, the modulated gradient model and the traditional Smagorinsky model use almost the same computational time (with a difference of less than $<0.1 \%$ ). This time is significantly shorter than the ones needed by the simulations with the Lagrangian scale-dependent dynamic model and the Lagrangian dynamic model, which take $31 \%$ and $20 \%$ more time, respectively. This comparison explicitly shows the low computational cost of the modulated gradient model compared with the Lagrangian scale-dependent dynamic and Lagrangian dynamic models. As mentioned in Section 1, this is due to the simplicity of the model as no test filtering or additional transport equation is needed.

\subsection{Mean velocity profiles}

The measured and simulated vertical profiles of the streamwise and vertical mean velocities (with grid resolution of $160 \times 40 \times 160$ ) are compared in Fig. 5. The general patterns observed in the measured velocity profiles are captured by all the models despite the fact that some differences are observed in the region above the block. Both experimental and LES results show the slow down of the ground-level streamwise velocity in the upstream region as the laminar inflow approaches the block. Moreover, due to the big recirculation formed above the block, negative streamwise velocity right above the block is observed in the experimental results.

Excellent agreement is found between the results of the modulated gradient model and the experiment. The wind velocities above the block and at the downstream region are well reproduced by the modulated gradient model. Other than the modulated gradient model, the results of the Lagrangian scale-dependent dynamic model also show good agreement with the measurements. However, minor discrepancies are observed above the block, where the simulated streamwise velocity recovers to its free-stream value at a lower height compared with the experiment. This implies that the big recirculation zone simulated by the Lagrangian scaledependent dynamic model is a bit thinner vertically than those measured in the experiment and simulated with the modulated gradient model. Simulations with the Lagrangian dynamic model and Smagorinsky model (with $C_{s}=0.1$ ) reproduce very similar mean wind profiles. Compared with the experimental data, the Lagrangian dynamic model and Smagorinsky model predict a smaller magnitude of the reverse mean flow above the block and at the downstream region. This is consistent with the results discussed in Section 3.1 that the big recirculation simulated by these two models is relatively shorter and weaker. For the vertical velocity profiles, all simulations give similar results, which compare well with the measurement.

It should be noted that in Fig. 5a, the thicknesses of the boundary layers developed above the ground upstream of the block and above the block are approximately equal to $0.1 \mathrm{~h}$. Comparing with the four grid resolutions tested in Section 3.1, the first grid points above the ground for the streamwise velocity are located at $\Delta_{z} / 2=0.025 h, 0.031 h, 0.042 h$ and $0.625 h$ for the grid resolutions of $200 \times 50 \times 200, \quad 160 \times 40 \times 160,120 \times 30 \times 120$ and $80 \times 20 \times 80$, respectively. Therefore, for the simulations with the two fine grid resolutions, there are at least two grid points near the ground surface or above the block are within the boundary layer $(<0.1 \mathrm{~h})$. In contrast, for the two coarse resolutions, there is only one grid point within the boundary layer, which is probably not enough to properly resolve the flow in those regions. This may explain the poor results obtained with these two resolutions in Section 3.1.

\subsection{Velocity variances}

\subsubsection{Contours}

The contours of the normalized resolved streamwise $\left(\left\langle\tilde{u}^{\prime} \tilde{u}^{\prime}\right\rangle / U_{0}^{2}\right)$, spanwise $\left(\left\langle\tilde{v}^{\prime} \tilde{v}^{\prime}\right\rangle / U_{0}^{2}\right)$ and vertical $\left(\left\langle\tilde{w}^{\prime} \tilde{w}^{\prime}\right\rangle / U_{0}^{2}\right)$ 

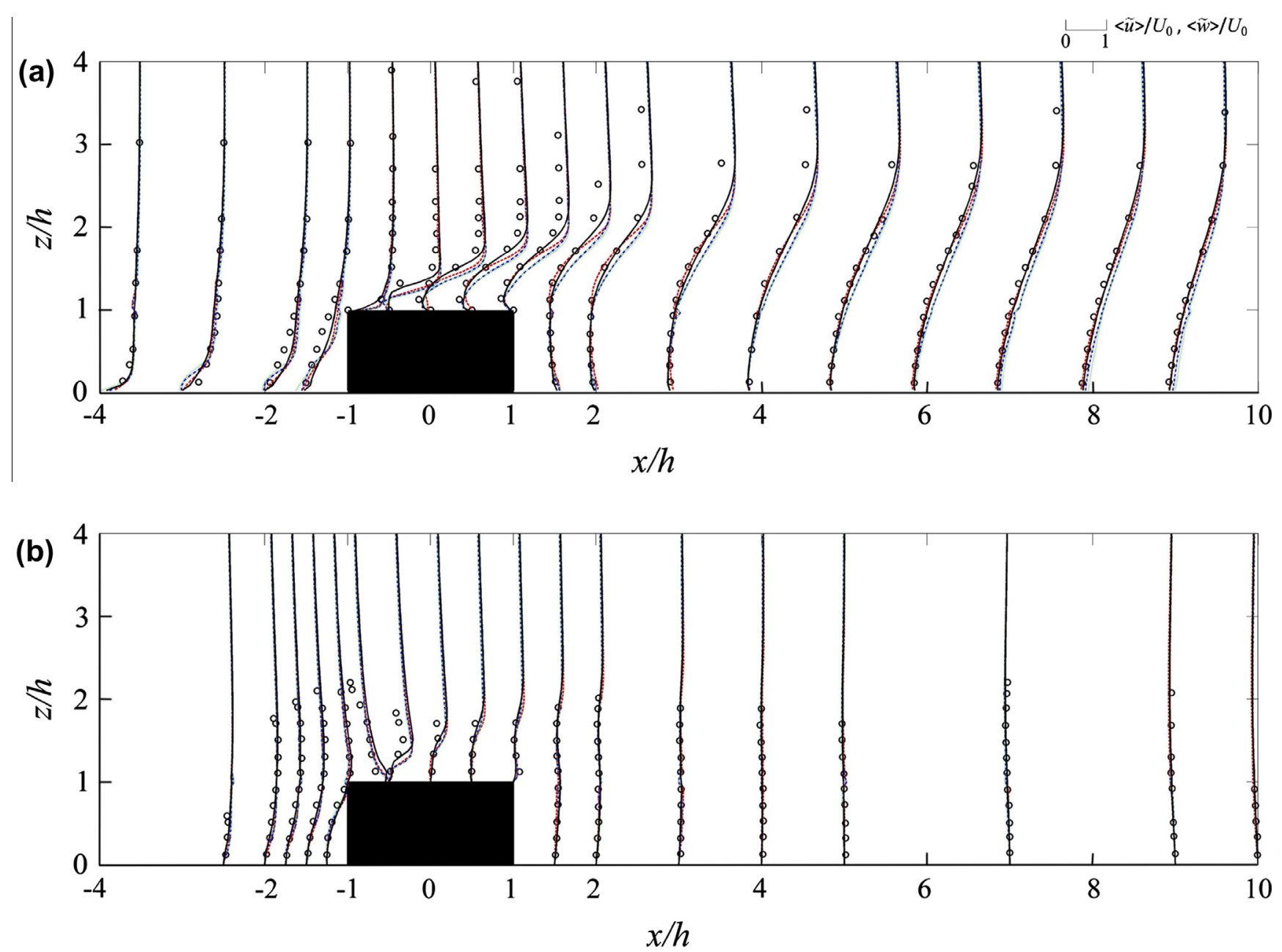

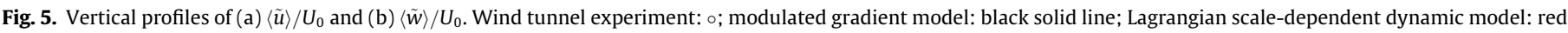

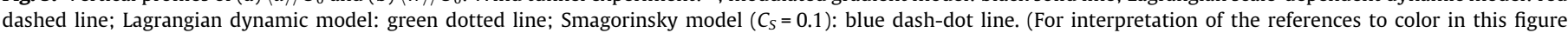
legend, the reader is referred to the web version of this article.)

velocity variances from the simulations with the four SGS models and a grid resolution of $160 \times 40 \times 160$ are shown in Fig. 6 . In all the contours, high values of velocity variances are observed in the region just above the block as turbulence is generated due to the strong shear produced when the incoming flow is deflected by the block. The turbulence generated above the block is transported by the mean wind and turbulent fluctuations to the neighbouring region, which increases the magnitudes of the velocity variances there. This TKE transport mechanism is discussed in more detail with the analysis of all the terms in the TKE budget equation presented in Section 3.3.3.

Discrepancies among the results of the SGS models are found above the block and at the downstream region. For the modulated gradient model, a region with high normalized streamwise velocity variance $\left(\left\langle u^{\prime} u^{\prime}\right\rangle / U_{0}^{2}\right)$ with magnitude approximately equal to 0.1 is found to begin just above the top upwind corner of the block and extend to the downstream position of $x=2 h$. For both the Lagrangian dynamic model and the Smagorinsky model, a region with maximum normalized streamwise velocity variance of around 0.1 is also found just downstream of the top upwind corner. This region has a larger extent than the one obtained with the modulated gradient model. For all the SGS models, the region of large velocity variances extends downwind to distances of more than $10 \mathrm{~h}$ from the block. Larger maximum value of the normalized streamwise velocity variance compared with other velocity components is also found.
For the spanwise and vertical velocity variances, differences in the locations of their maximum values are found among the different SGS models. For the modulated gradient model, the maximum spanwise and vertical velocity variances are both found at a location about $1 h$ above the top downwind corner of the block, and they have a normalized value of about 0.07. In turn, for the Lagrangian scale-dependent dynamic and the Lagrangian dynamic models, the maximum values of spanwise and vertical velocity variances appear at a further downstream position of about $x / h=8$ and 5 , respectively. Larger maximum value of the normalized spanwise velocity variance of about 0.08 than that of the vertical velocity variance of about 0.07 are also found in the results of these two models. For the Smagorinsky model, the maximum spanwise and vertical velocity variances regions extend from above the block to downstream of the block. Larger maximum value of the normalized spanwise velocity variance than the vertical velocity variance is also found.

\subsubsection{Vertical profiles}

The measured and simulated profiles of the streamwise, spanwise and vertical velocity variances are shown in Fig. 7. The simulation with the modulated gradient model shows the best overall agreement with the measurements. In particular, good agreement of the normalized velocity variances above the block and at the 


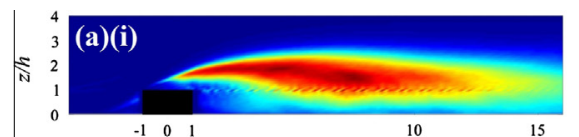

$x / h$

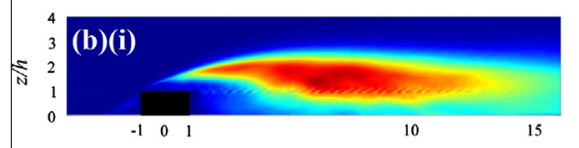

$x / h$

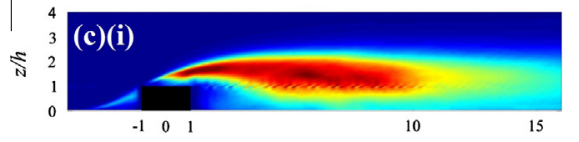

$x / h$

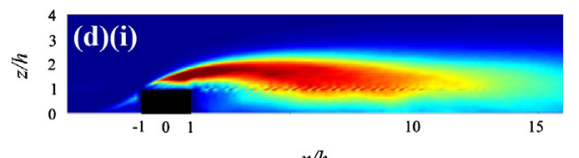

$x / h$

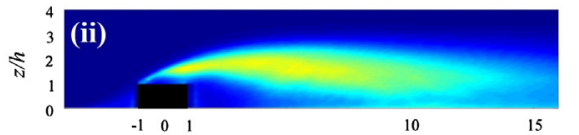

$x / h$

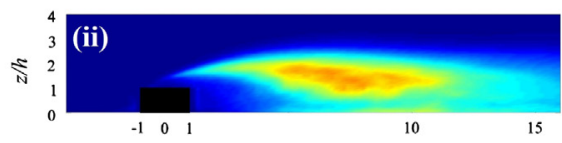

$x / h$

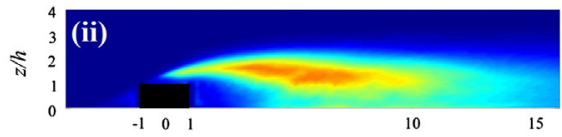

$x / h$

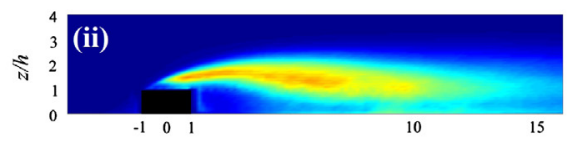

$x / h$

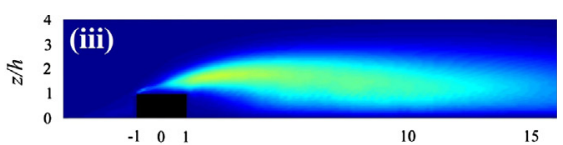

$x / h$

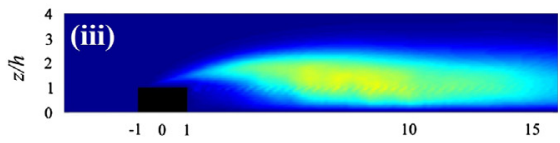

$x / h$

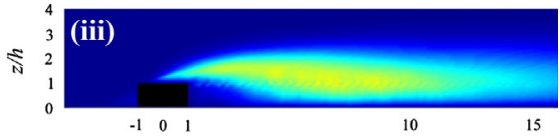

$x / h$

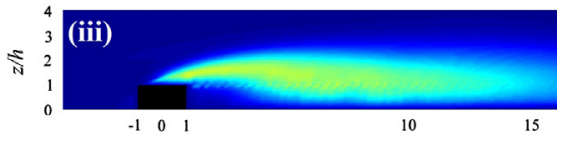

$x / h$

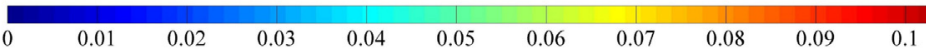

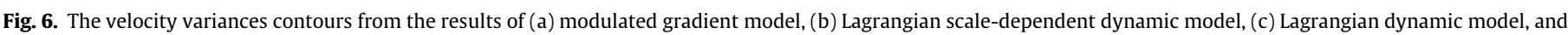
(d) Smagorinsky model $\left(C_{S}=0.1\right)$ with (i) $\left\langle\tilde{u}^{\prime} \tilde{u}^{\prime}\right\rangle / U_{0}^{2}$, (ii) $\left\langle\tilde{v}^{\prime} \tilde{v}^{\prime}\right\rangle / U_{0}^{2}$ and (iii) $\left\langle\tilde{w}^{\prime} \tilde{w}^{\prime}\right\rangle / U_{0}^{2}$.

downstream position with the measurement are observed despite the fact that some over-predictions of the streamwise and vertical velocity variances are found in the profiles just behind the block. Different from the results of the modulated gradient model, the Lagrangian scale-dependent dynamic model gives very good prediction of the three velocity variances in the regions downstream of the block. However, in the region above the block, the values of the velocity variances are underestimated. This is probably due to the uncertainty caused when performing the primary and secondary test filtering in the SGS model at the small region above the block where strong flow separation and recirculation occur. For the Lagrangian dynamic model and the Smagorinsky model, similar trends are obtained in the profiles of the simulated velocity variances. At the region above the block, the two models are able to reproduce roughly the same magnitudes of velocity variances as in the measurements. However, the predicted peak values of the velocity variances profiles are found at a lower vertical position than those in the experiment. Moreover, the velocity variances are over-predicted just downstream of the block, with magnitudes similar to those obtained with the modulated gradient model. The vertical velocity variance is also under-predicted further downstream $(x / h=10)$.

The better performance of the modulated gradient model compared with the other three eddy-viscosity-based models is related to the different formulation of the models, which gives the modulated gradient model a better capacity to model flow anisotropy. As discussed in Lu and Porté-Agel (2010), the eddy-viscosity model uses a scalar eddy viscosity (the same value for all directions), which hinders its ability to account for strong flow anisotropy, especially on coarse grids. In contrast, the modulated gradient model uses the gradient tensor (which can be obtained by Taylor's expansion of the SGS stress) to determine the structure (relative magnitude of the different components) of the SGS stress tensor. In simulations of a neutral atmospheric boundary layer flow, Lu and Porté-Agel (2010) demonstrated the better performance of the modulated gradient model in resolving the highly anisotropic near-surface flow compared with the results of the traditional Smagorinsky model. This is consistent with the current finding that the results of the modulated gradient model show the best overall agreement with the experimental data among the four SGS models tested.

\subsubsection{Turbulence kinetic energy (TKE) budget}

In order to better understand the TKE production and transport mechanisms in the flow past the 2D block, the TKE budget is considered using the LES results. The TKE budget equation for a neutrally-stratified incompressible flow that is homogeneous in the spanwise direction can be written as

$$
\begin{aligned}
\underbrace{\frac{\partial\langle k\rangle}{\partial t}=}_{\mathrm{S}} & \underbrace{-\left(\left\langle\tilde{u}_{1}^{\prime} \tilde{u}_{3}^{\prime}\right\rangle+\left\langle\tau_{13}\right\rangle\right)\left(\frac{\partial\left\langle\tilde{u}_{1}\right\rangle}{\partial x_{3}}+\frac{\partial\left\langle\tilde{u}_{3}\right\rangle}{\partial x_{1}}\right)+\left(\left\langle\tilde{u}_{3}^{\prime 2}\right\rangle-\left\langle\tilde{u}_{1}^{\prime 2}\right\rangle+\left\langle\tau_{33}\right\rangle-\left\langle\tau_{11}\right\rangle\right) \frac{\partial\left\langle\tilde{u}_{1}\right\rangle}{\partial x_{1}}}_{\mathrm{P}} \\
& \underbrace{-\left\langle\tilde{u}_{j}\right\rangle \frac{\partial\langle k\rangle}{\partial x_{j}}}_{\mathrm{A}} \underbrace{-\frac{1}{2} \frac{\partial\left\langle\tilde{u}_{i}^{\prime} \tilde{u}_{i}^{\prime} \tilde{u}_{j}^{\prime}\right\rangle}{\partial x_{j}}}_{\mathrm{T}_{t}}+\mathrm{R},
\end{aligned}
$$

where $\langle k\rangle=\frac{1}{2}\left\langle u_{i}^{\prime} u_{i}^{\prime}\right\rangle$ is the TKE, $t$ is the time, $\mathrm{S}$ is the storage term, A is the advection term, $\mathrm{P}$ is the mechanical (shear) production term, $\mathrm{T}_{t}$ is the turbulence transport term, and $\mathrm{R}$ is the residual term. The storage term $S$ represents the change of TKE with time and should be equal to zero for flow in quasi-steady state. The results of the modulated gradient model are used to compute the values of the TKE budget terms which are shown in Fig. 8 .

As reflected by the mechanical production term $(\mathrm{P})$, substantial TKE production is associated with the high shear region that starts at the upwind edge of the block and extends upward and downwind as the flow is deflected around the block. The region with maximum TKE production is at a position of about $0.5 h$ above the upper surface of the block. The high level of maximum TKE production above the block is related to the recirculation zone formed just above the block due to the flow separation occurring near the top upwind corner of the block. This is consistent with the results of velocity variances in Figs. 6 and 7, which show the high values of velocity variances above the block, with a layer of low velocity variances below. The high TKE production region extends further downstream. Part of the shear-generated TKE is transported by 

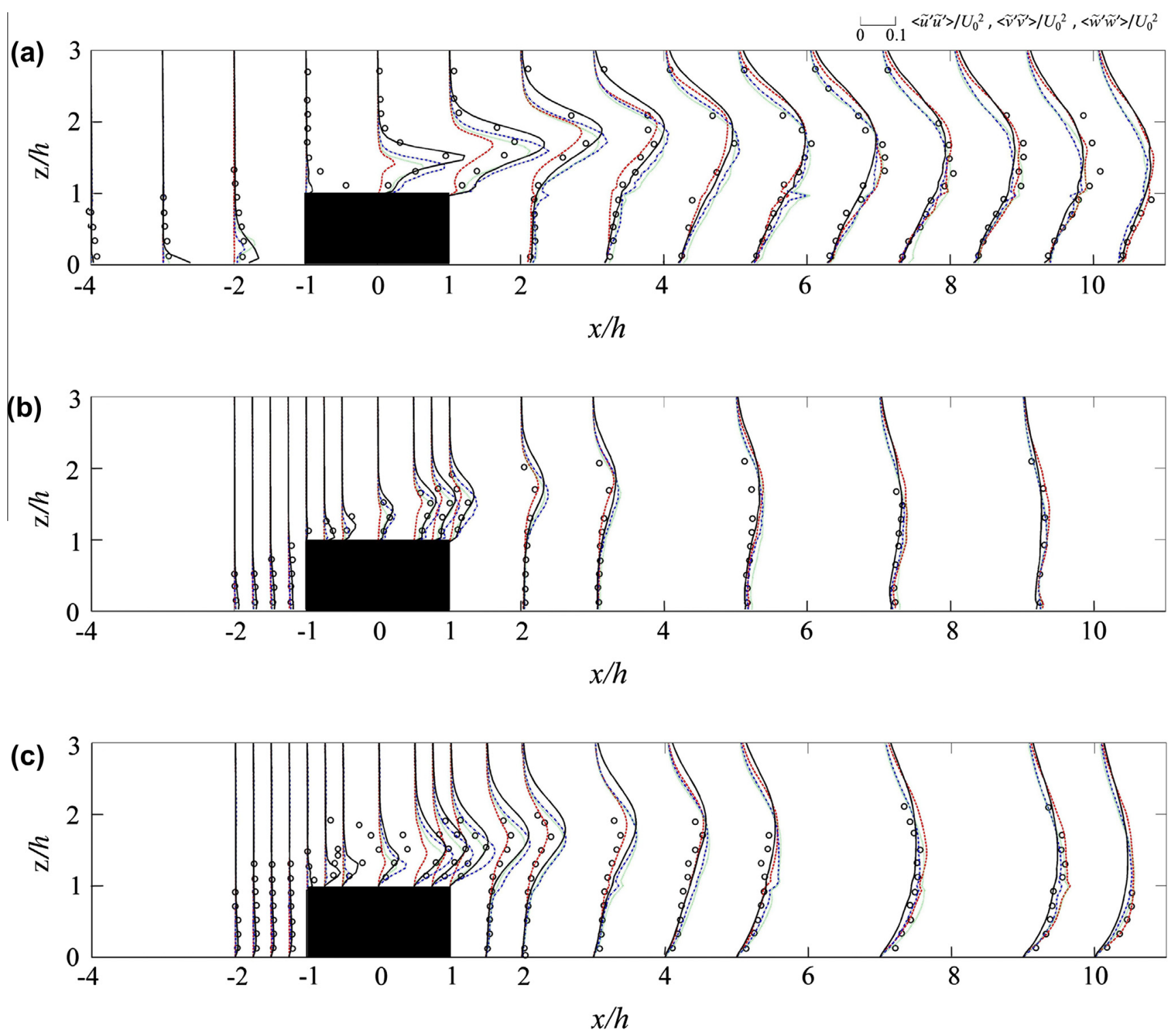

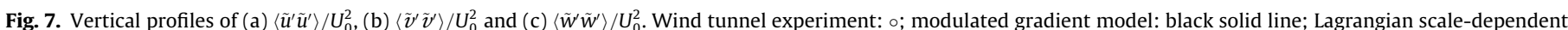

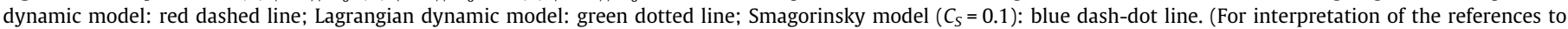
color in this figure legend, the reader is referred to the web version of this article.)
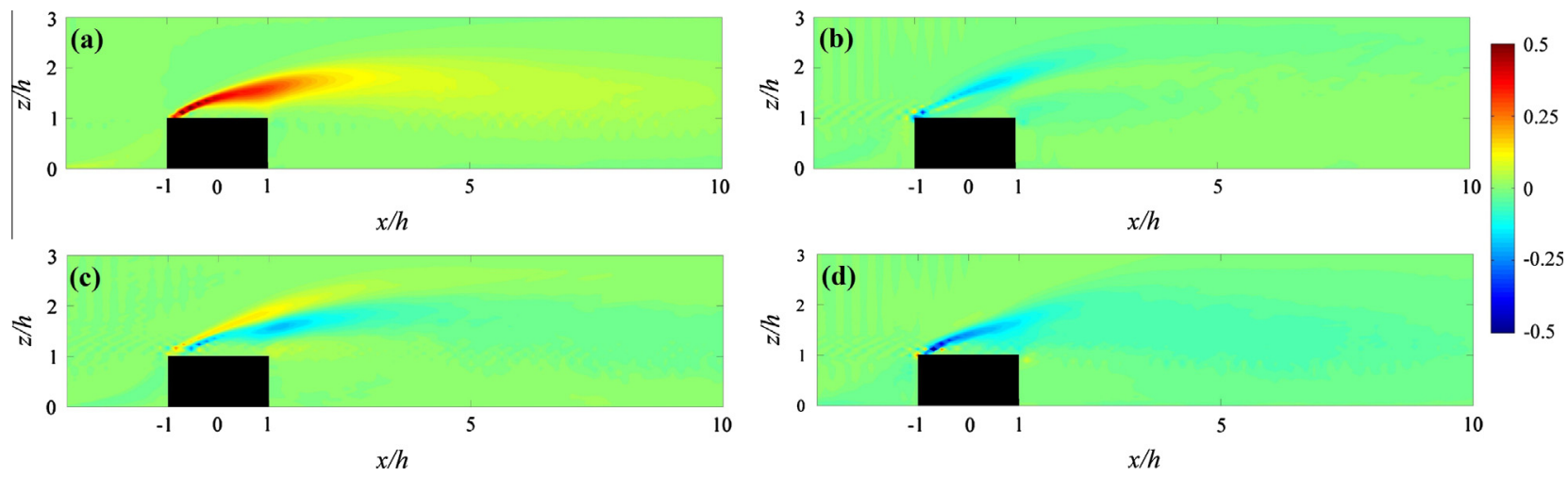

Fig. 8. Contours of normalized (by $U_{0}^{3} / h$ ) TKE budget terms: (a) Mechanical (shear) production, (b) advection (c), turbulent transport, and (d) residual.

the mean wind as demonstrated by the negative value of the advection term above the block. Moreover, as reflected by the turbulence transport term $\left(\mathrm{T}_{t}\right)$ distribution, TKE is transported by turbulence upward and away from the high-shear production region. The residual term is calculated from the other terms by enforcing the condition of $S=0$. It includes the contribution of 
viscous dissipation, pressure-correlation, and the SGS turbulence transport. As shown in Fig. 8, the residual term has a significant contribution and mainly acts as a sink term.

\section{Conclusions}

Large-eddy simulations of flow past a 2D block were performed to evaluate the traditional Smagorinsky model, the Lagrangian dynamic model, the Lagrangian scale-dependent dynamic model and the modulated gradient model by comparing the simulation results with wind tunnel experimental data (Baker, 1977; Moss and Baker, 1980). An immersed boundary (IB) method was implemented to model the boundaries of the 2D block in the Cartesian grid. A uniform grid was used and four different resolutions $N_{x} \times N_{y} \times N_{z}=200 \times 50 \times 200, \quad 160 \times 40 \times 160$, $120 \times 30 \times 120$ and $80 \times 20 \times 80$ were considered for all SGS models. These correspond to a number of grid points $\left(n_{x} \times n_{z}\right)=$ $16 \times 20,13 \times 16,10 \times 12$ and $6 \times 8$ covering the block, respectively.

Our results show that the modulated gradient model has the best overall performance among the four subgrid-scale (SGS) models. Grid independent results are achieved at the grid resolution of $160 \times 40 \times 160$ as nearly the same flow statistics are obtained as those from the fine grid resolution of $200 \times 50 \times 200$. Excellent agreement with wind tunnel experimental results is found in the flow recirculation pattern, the reattachment position and the vertical profiles of mean velocities and velocity variances. This suggests that accurate mean wind and velocity variances can be obtained by the modulated gradient model with $13 \times 16$ grid points across the block. The Lagrangian scale-dependent dynamic model has the second best overall performance. Despite the fact that some discrepancies with the experimental results are observed in the velocity variances profiles, the general features of the flow are reproduced. Rather poor prediction of the flow recirculation pattern is observed with the Lagrangian dynamic model and the Smagorinsky model. In particular, for the Smagorinsky model, the simulation results are found to be very sensitive to the choice of the Smagorinsky coefficient $C_{s}$ as demonstrated by the large differences found in the results between the simulations with $C_{s}=0.1$ and 0.2. In addition, the turbulence kinetic energy (TKE) budget of the flow was investigated using the LES results. Strong shear production is found as the incoming flow is deflected by the block. The TKE produced is transported horizontally and vertically respectively by mean wind and turbulence.

This is the first time that the modulated gradient model is validated in simulations of a recirculating flow, and excellent results are obtained compared with experimental data. The advantage of the modulated gradient model is that it is simple and computationally inexpensive (comparable with the standard Smagorinsky model) as no additional transport equations or test filtering operations is required. Therefore, the remarkable performance of the model found in this study make it an interesting simple alternative to eddy-viscosity models for LESs of high-Reynolds-number recirculating flows over complex terrain. Future research will extend the model validation to different terrain geometries (urban canopies and/or topography) and include scalar transport for different thermal stratification conditions.

\section{Acknowledgements}

This research was supported by the Swiss National Science Foundation (Grant 200021-132122). Computing resources were provided by the Minnesota Supercomputing Institute (MSI) and the Swiss National Supercomputing Centre (CSCS) under Project ID s306.

\section{References}

Abkar, M., Porté-Agel, F., 2012. A new boundary condition for large-eddy simulation of boundary-layer flow over surface roughness transitions. Journal of Turbulence 13, 1-18.

Albertson, J.D., 1996. Large eddy simulation of land-atmosphere interaction. PhD Thesis, University of California Davis.

Baker, S., 1977. Regions of recirculating flow associated with two-dimensional steps. PhD Thesis, University of Surrey.

Businger, J.A., Wyngaard, J.C., Izumi, Y., Bradley, E.F., 1971. Flux-profile relationships in the atmospheric surface layer. Journal of the Atmospheric Sciences 28, 181189.

Cai, X.-M., 1999. Large-eddy simulation of the convective boundary layer over an idealized patchy urban surface. Quarterly Journal of the Royal Meteorological Society $125,1427-1444$.

Castro, I.P., Robins, A.G., 1977. The flow around a surface-mounted cube in uniform and turbulent streams. Journal of Fluid Mechanics 79, 307-335.

Chamorro, L., Porté-Agel, F., 2010. Wind-tunnel study of surface boundary conditions for large-eddy simulation of turbulent flow past a rough-tosmooth surface transition. Journal of Turbulence 11, 1-17.

Fang, J., Diebold, M., Higgins, C., Parlange, M., 2011. Towards oscillation-free implementation of the immersed boundary method with spectral-like methods. Journal of Computational Physics 230, 8179-8191.

Germano, M., Piomelli, U., Moin, P., Cabot, W.H., 1991. A dynamic subgrid-scale eddy viscosity model. Physics of Fluids 3, 1760-1765.

Hinze, J.O., 1975. Turbulence, second ed. McGraw-Hill, 790 pp.

Hunt, J.C.R., Kawai, H., Ramsey, S.R., Pedrizetti, G., Perkins, R.J., 1990. A review of velocity and pressure fluctuations in turbulent flows around bluff bodies. Journal of Wind Engineering and Industrial Aerodynamics 35, 49-85.

Hussein, H.J., Martinuzzi, R.J., 1996. Energy balance for turbulent flow around a surface mounted cube placed in a channel. Physics of Fluids 8, 764-780.

Krajnović, S., 2009. Large eddy simulation of flows around ground vehicles and other bluff bodies. Philosophical Transactions of The Royal Society A 367, 2917 2930.

Krajnović, S., Davidson, L., 2002. Large-eddy simulation of the flow around a bluff body. AIAA Journal 40 (5), 927-936.

Lim, H.C., Thomas, T.G., Castro, I.P., 2009. Flow around a cube in a turbulent boundary layer: LES and experiment. Journal of Wind Engineering and Industrial Aerodynamics 97, 96-109.

Lu, H., Porté-Agel, F., 2010. A modulated gradient model for large-eddy simulation: application to a neutral atmospheric boundary layer. Physics of Fluids 22 (015109).

Marusic, I., Kunkel, G.J., Porté-Agel, F., 2001. Experimental study of wall boundary conditions for large-eddy simulation. Journal of Fluid Mechanics 446, 309-320.

Meneveau, C., Katz, J., 2000. Scale-invariance and turbulence models for large-eddy simulation. Annual Review of Fluid Mechanics 32, 1-32.

Meneveau, C., Lund, T.S., Cabot, W.H., 1996. A Lagrangian dynamic subgrid-scale model of turbulence. Journal of Fluid Mechanics 319, 353-385.

Mittal, R., Iaccarino, G., 2005. Immersed boundary methods. Annual Review of Fluid Mechanics 37, 239-261.

Moss, W.D., Baker, S., 1980. Re-circulating flows associated with two-dimensional steps. Aeronautical Quaterly 31, 151-172.

Murakami, S., Mochida, A., Hibi, K., 1987. Three-dimensional numerical simulation of air flow around a cubic model by means of large eddy simulation. Journal of Wind Engineering and Industrial Aerodynamics 25, 291-305.

Orszag, S.A., Pao, Y.-H., 1975. Numerical computation of turbulent shear flows. Advances in Geophysics 18 A, 225-236.

Pope, S.B., 2000. Turbulent Flows. Cambridge University Press, 771 pp.

Porté-Agel, F., 2004. A scale-dependent dynamic model for scalar transport in largeeddy simulations of the atmospheric boundary layer. Boundary-Layer Meteorology 112, 81-105.

Porté-Agel, F., Meneveau, C., Parlange, M.B., 2000. A scale-dependent dynamic model for large-eddy simulation: application to a neutral atmospheric boundary layer. Journal of Fluid Mechanics 415, 261-284.

Porté-Agel, F., Wu, Y.T., Lu, H., Conzemius, R.J., 2011. Large-eddy simulation of atmospheric boundary layer flow through wind turbunes and wind farms. Journal of Wind Engineering and Industrial Aerodynamics 99, 154-168.

Sagaut, P., 2005. Large eddy simulation for incompressible flows: an introduction third ed. Springer, 556 pp.

Shah, K.B., Ferziger, J.H., 1997. A fluid mechanicians view of wind engineering: large eddy simulation of flow past a cubic obstacle. Journal of Wind Engineering and Industrial Aerodynamics, 211-224.

Smagorinsky, J.S., 1963. General circulation experiments with the primitive equations. Part 1. Basic experiments. Monthly Weather Review 91, 99-164.

Stoll, R., Porté-Agel, F., 2006a. Dynamic subgrid-scale models for momentum and scalar fluxes in large-eddy simulations of neutrally stratified atmospheric boundary layers over heterogeneous terrain. Water Resources Research 42, W01409.

Stoll, R., Porté-Agel, F., 2006b. Effect of roughness on surface boundary conditions for large-eddy simulation. Boundary-Layer Meteorology 118, 169-187.

Stoll, R., Porté-Agel, F., 2008. Large-eddy simulation of the stable atmospheric boundary layer using dynamic models with different averaging schemes. Boundary-Layer Meteorology 126, 1-28.

Suksangpanomrung, A., Djilali, N., Moinat, P., 2000. Large-eddy simulation of separated flow over a bluff rectangular plate. International Journal of Heat and Fluid Flow 21, 655-663. 
Tseng, Y.-H., Meneveau, C., Parlange, M.B., 2006. Modeling flow around bluff bodies and predicting urban dispersion using large eddy simulation. Environmental Science and Technology 40, 2653-2662.

Voller, V.R., Porté-Agel, F., 2002. Moore's law and numerical modeling. Journal of Computational Physics 179, 698-703.

Wan, F., Porté-Agel, F., 2011a. Large-eddy simulation of stably-stratified flow over a steep hill. Boundary-Layer Meteorology 138 (3), 367-384.

Wan, F., Porté-Agel, F., 2011b. A large-eddy simulation study of turbulent flow over multiscale topography. Boundary-Layer Meteorology 141 (2), 201-217.

Wan, F., Porté-Agel, F., Stoll, R., 2007. Evaluation of dynamic subgrid-scale models in large-eddy simulations of neutral turbulent flow over a two-dimensional sinusoidal hill. Atmospheric Environment 41, 2719-2728.
Wu, Y.T., Porté-Agel, F., 2011. Large-eddy simulation of wind-turbine wakes: evaluation of turbine parametrisations. Boundary-Layer Meteorology 138, 345-366.

Wu, Y.T., Porté-Agel, F., 2012. Atmospheric turbulence effects on wind-turbine wakes: an LES study. Energies 5, 5340-5362.

Wu, Y.T., Porté-Agel, F., 2013. Simulation of turbulent flow inside and above wind farms: model validation and layout effects. Boundary-Layer Meteorology 146, 181-205.

Yakhot, A., Liu, H., Nikitin, N., 2006. Turbulent flow around a wall-mounted cube: a direct numerical simulation. International Journal of Heat and Fluid Flow 27 994-1009. 